\title{
SEMI-SUPERVISED LEARNING OF BRAIN FUNCTIONAL NETWORKS
}

\author{
Yuhui Du ${ }^{a, b}$, Jing Sui ${ }^{a}$, Qingbao Yu ${ }^{a}$, Hao He ${ }^{a, c}$, Vince D. Calhoun ${ }^{a, c}$ \\ ${ }^{a}$ The Mind Research Network, Albuquerque, NM, USA \\ ${ }^{b}$ School of Information and Communication Engineering, North University of China, Taiyuan, China \\ ${ }^{c}$ Department of Electrical and Computer Engineering, University of New Mexico, Albuquerque, NM, \\ $U S A$
}

\begin{abstract}
Identification of subject-specific brain functional networks of interest is of great importance in fMRI based brain network analysis. In this study, a novel method is proposed to identify subject-specific brain functional networks using a graph theory based semi-supervised learning technique by incorporating not only prior information of the network to be identified as similarly used in seed region based correlation analysis (SCA) but also background information, which leads to robust performance for fMRI data with low signal noise ratio (SNR). Comparison experiments on both simulated and real fMRI data demonstrate that our method is more robust and accurate for identification of known brain functional networks than SCA, blind independent component analysis (ICA), and clustering based methods including Ncut and Kmeans.
\end{abstract}

Index Terms- semi-supervised learning, brain functional network, independent component analysis, clustering

\section{INTRODUCTION}

FMRI imaging studies have revealed the presence of functional connectivity among anatomically separated brain regions and a functional network typically consists of regions with coherent functional fluctuations. The identification of functional networks from fMRI data are typically implemented by either hypothesis based methods or data driven methods [1]. The most common hypothesis based method is seed region based correlation analysis (SCA) method [2], which identifies brain regions with functional signals highly correlated with the seed region's functional signal. SCA has been widely applied in brain functional network studies due to its simplicity. However, the resulting network from SCA is the seed region centered network, consequently is limited by selection of the seed region. In addition, it is often difficult to determine proper thresholds for getting subject-specific networks due to the inter-subject variability.

Several data driven methods have also been proposed for detecting functional networks, such as independent component analysis (ICA) [3-5] and clustering based approaches [6]. ICA, especially spatial ICA, extracts from spatiotemporal fMRI data spatially independent components (ICs), which are interpreted as different functional networks. For ICA, it typically needs a good estimation of the number of ICs to be computed for obtaining brain functional networks robustly. Clustering voxelwise functional signals is a potentially powerful approach for exploring functional networks, and clustering algorithms such as Kmeans, hierarchical clustering [7], normalized cut (Ncut) [6], support vector clustering
(SVC) [8], have been adopted in fMRI studies. However, it is difficult to determine an appropriate number of clusters.

To overcome the limitations of existing methods, we propose a graph theory based semi-supervised learning approach which extracts subject-specific functional networks from fMRI data with guidance of reliable prior knowledge. Recently, a semi-supervised learning technique has been proposed to obtain subject-specific and functional consistent regions of interest (ROIs) that are taken as nodes of network for correlation analysis [9]. In this paper, functional network is extracted from the whole brain data, and seeds are automatically computed using the individual data based on prior information.

\section{METHODS}

The proposed method extracts subject-specific functional networks based on individual data and reliable prior information. Firstly, seeds of functional network and background are automatically determined based on prior information. Then a graph theory based semi-supervised learning method is applied to extract functional network based on those seeds.

2.1. Semi-supervised learning of functional network

In our method, graph theory based technique is used to model 3D brain image as a graph $G(V, E)$, where each node $V$ corresponds to a voxel of the $3 \mathrm{D}$ image and each edge $E$ connecting a pair of voxels reflects the similarity of BOLD time series of the pair of voxels. Based on obtained seeds, graph theory based semisupervised learning method is applied to extract functional network of interest though assigning different labels to voxels, some belong to the functional network, and others are assigned to the background.

Most semi-supervised learning methods define a continuous classification function $F$ that is estimated on the graph to minimize a cost function. The cost function enforces a tradeoff between the smoothness of the function on the graph of both labeled and unlabeled data and the accuracy of the function at fitting the label information for the initial seeds. A survey for semi-supervised learning is available in a paper [10], which involves the mincuts method [11], the Gaussian fields and harmonic function method [12], the local and global consistency method (LGC) [13], and the graph transduction via alternating minimization method [14].

Given initial labels of a few voxels, labels of unlabeled voxels can be predicted by exploiting the similarity between voxels. In the LGC method, the labeling problem can be solved by minimizing a cost function within a regularization framework[13]

$$
Q(F)=\sum_{i, j}^{n} w_{i j}\left\|\frac{F_{i}}{\sqrt{D_{i i}}}-\frac{F_{j}}{\sqrt{D_{j j}}}\right\|^{2}+\mu \sum_{i=1}^{n}\left\|F_{i}-Y_{i}\right\|^{2},
$$


where $w_{i j}$ is the edge weight between nodes $i$ and $j, F_{i}$ is the label vector of the node $i, D_{i i}=\sum_{j=1}^{n} w_{i j}$ is the degree of the node $i, Y_{i}$ is the initial label vector of the node $i$, and $n$ is the number of nodes. The first term of eqn.(1) is a local consistency constraint to encourage similar voxels to have similar labels, and the second term measures the consistency between the labeling result and the initial labeling information. These two terms are balanced by the parameter $\mu$ to achieve labeling with local and global consistency. The eqn.(1) also can be approximated in the matrix form as:

$$
Q(F)=\operatorname{tr}\left(F^{T}(I-S) F+\mu\left(F-Y_{i n i}\right)^{T}\left(F-Y_{i n i}\right)\right),
$$

where $I$ is an identity matrix, $S=D^{-1 / 2} W D^{1 / 2}$ is the normalized edge weight matrix, $W=\left\{w_{i j}\right\}, F \in R^{n \times c}$ is the classification function matrix, $Y_{i n i} \in R^{n \times c}$ is the initial label matrix, and $c$ is the number of clusters. In this paper, $c=2$ for partitioning the network to be identified and the background. The minimization of $Q(F)$ can be achieved by an iterative procedure that has been demonstrated to converge to the optimal solution

$$
F^{m+1}=(1-\alpha) S F^{m}+\alpha Y_{i n i},
$$

where $F^{m}$ is the updated label information at the $m^{\text {th }}$ iteration, $F^{0}$ is equivalent to $Y_{\text {ini }}$, and $\alpha(0<\alpha<1)$ is a parameter related to $\mu$. $\alpha$ was set to 0.9 in this paper. This iterative procedure can be regarded as propagation of label information. At each iteration, every voxel absorbs the label information from other voxels and retains partial label information of its initial state. The label information is updated until convergence and each voxel is assigned to the class from which it receives the most information.

\subsection{Edge weight measurement between voxels}

For extracting functional networks, affinity of pair-wise voxels is measured by Pearson correlation of BOLD time series in this paper since that the temporal correlation between BOLD signal is often used to measure functional connectivity. Particularly, the edge weight between voxels $i$ and $j$ is computed by

$$
w_{i j}=\operatorname{corr}\left(T_{i}, T_{j}\right) \text {, }
$$

where $T_{i}$ refers to the BOLD time series of the voxel $i, \operatorname{corr}(\because ;)$ denotes computing Pearson correlation coefficient. After obtaining the affinity matrix, sparsity of graph is obtained through constructing a k-nearest graph considering that sparsity is important to ensure a graph-based algorithm efficient and robust to noise. $\mathrm{K}$ is used to denote the parameter in the k-nearest graph construction.

\subsection{Determination of reliable seeds}

Reliable seeds are significant for robust functional network. In this study, firstly, few reliable voxels that belong to the functional network based on prior anatomical information are selected as objective seeds. And then, voxels that have negative temporal correlation with the mean BOLD time series of those objective seeds are automatically computed as seeds of the background.

\section{EXPERIMENT RESULTS}

The proposed method was validated using simulated data and real fMRI data. Experiments using simulated data of different signal noise ratios (SNRs) were performed to evaluate the method with respect to the spatial feature, the signal property and the network property of the extracted functional network, comparing with SCA, ICA, Ncut and Kmeans methods. Experiments using resting-state fMRI data of 106 healthy subjects collected at 5 sites, including Bangor (19 subjects), Berlin_Margulies (26 subjects), Leiden_2200 (19 subjects), NewYork_b (20 subjects), and Oxford (22 subjects) were performed by the proposed method for identifying the default mode network (DMN) and motor network, and then the reproducibility and reliability of those networks across subjects of different sites were investigated.

\subsection{Experiment using simulated data}

Simulated data was generated as follows. We used preprocessed resting-state fMRI data as baseline data, and then added simulated signals for voxels of the baseline data. The added signal for each voxel of the simulated network (see Fig. 1(A)) was a mixture of sinusoidal function with 100 time points and white Gaussian noise. The added signal in other regions was a mixture of sinusoidal function with a random phase difference and white Gaussian noise. For evaluating the robustness of algorithms, different SNRs including 5, 4, 3, 2 and 1 were used. Here, $S N R=\frac{\operatorname{var}(\text { signal })}{\operatorname{var}(\text { noise })}$. One slice of the simulated data with SNR as 1 is shown in Fig. 1(B).
(A)

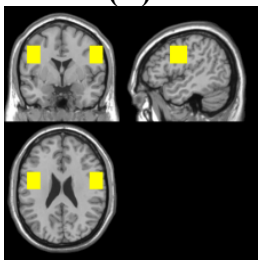

(B)

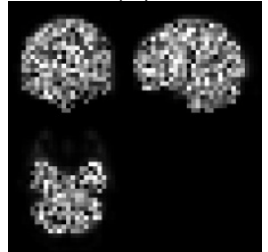

Fig. 1. Simulated functional network. (A) Regions of the simulated network. (B) One slice of the simulated 3D image with SNR as 1.

Using the simulated data, our method, SCA, ICA, Ncut, and Kmeans were evaluated according to the spatial feature, the signal property, and the network property of the extracted functional network. For the proposed method, four adjacent seeds from one block of the network were set as the objective seeds, and voxels that have negative correlation with the mean signal of the objective seeds were automatically obtained as the background seeds. Different $\mathrm{K}$ for constructing k-nearest graph were evaluated. For SCA, mean signal of the objective seeds selected in our method was used as representative signal for correlation analysis. After obtaining the correlation value for each voxel, the functional network was obtained by thresholding these correlation values using different thresholds ranging from 0.3 to 0.7 . For ICA, different IC numbers ranging from 5 to 30 and thresholds including 2 and 3 were applied. For Ncut and Kmeans, different cluster numbers ranging from 2 to 6 were used. While using ICA, Ncut, and Kmeans, the most similar IC or cluster according to the spatial similarity to the simulated network was selected for extracting the network. Subsequently, the spatial feature of the extracted network was measured by the agreement between the identified network and the simulated network. Agreement $=\frac{|A \cap B|}{|A \cup B|}$, where $A$ and $B$ respectively denote the identified and simulated network. The signal property was computed by the mean signal in the extracted network regions. The network property was represented by the mean of pair-wise correlations of BOLD in voxles of network regions. The signal property and the network property of the simulated network were also computed for comparison purpose.

Fig. 2 shows the networks extracted from different methods under the case of $\mathrm{SNR}=1$. For comprehensive evaluation, Fig. 3 demonstrates the spatial property of extracted network under different SNRs obtained from our method with different K, SCA with different thresholds, ICA with different IC numbers and thresholds, as well as Ncut and Kmeans with different cluster numbers. These results demonstrate that our method had higher accuracy and was more robust to SNR than other methods since the reliable prior information was used and global similarity of all voxels was considered under a graph theory based framework. 
The signal property and the network property of the extracted network are shown in Fig. 4 and Fig. 5, respectively. Results show that those measures from our method were closer to that of the simulated network, which indicates that our method is more robust and accurate for identification of this functional network than SCA, ICA, and clustering based methods including Ncut and Kmeans.

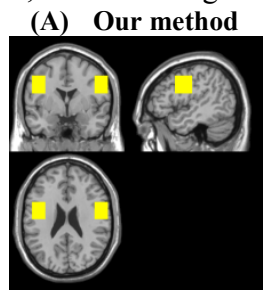

(D) ICA

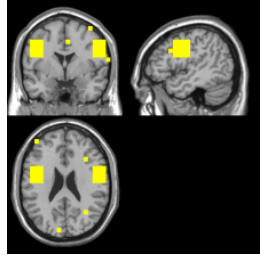

(B) SCA

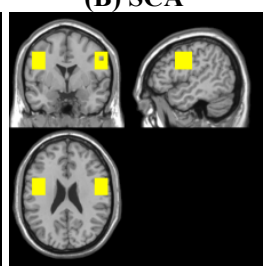

(E) Ncut

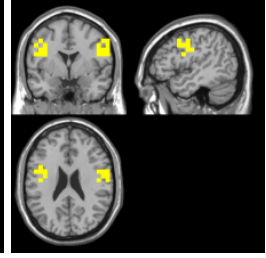

(C) ICA

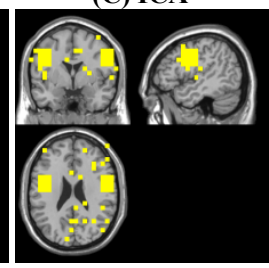

(F) Kmeans

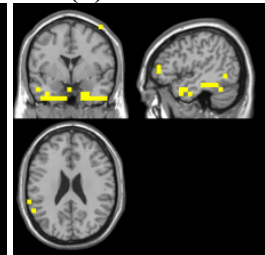

Fig. 2 The extracted network under the case of $\mathrm{SNR}=1$, obtained from (A) our method with $\mathrm{K}$ as 60 , (B) SCA with threshold as 0.5 , (C) ICA with IC number as 20 and threshold as 2, (D) ICA with IC number as 20 and threshold as 3, (E) Ncut with cluster number as 4, and (F) Kmeans with cluster number as 4.

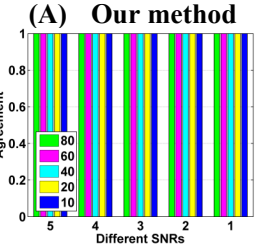

(D) ICA

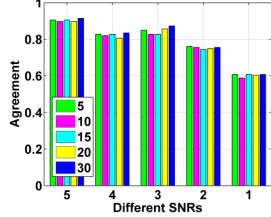

(B) $\mathrm{SCA}$

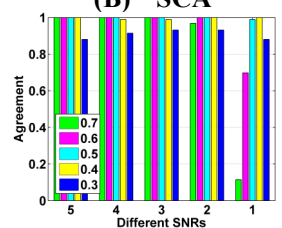

(E) Ncut

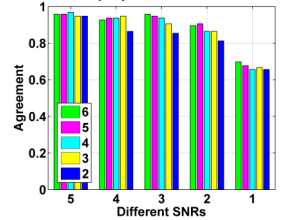

(C) ICA

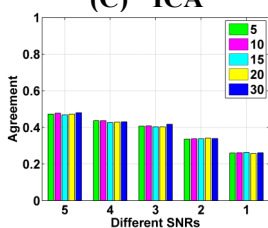

(F) Kmeans

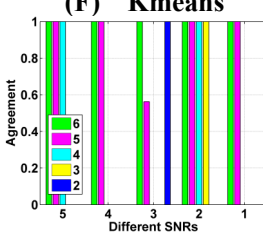

Fig. 3 Spatial property under different SNRs, obtained using (A) our method with different K, (B) SCA with different thresholds, (C) ICA with threshold as 2 and different IC numbers, (D) ICA with threshold as 3 and different IC numbers, (E) Ncut with different cluster numbers, and (F) Kmeans with different cluster numbers. Different colors denote different parameters.
(A) Our method

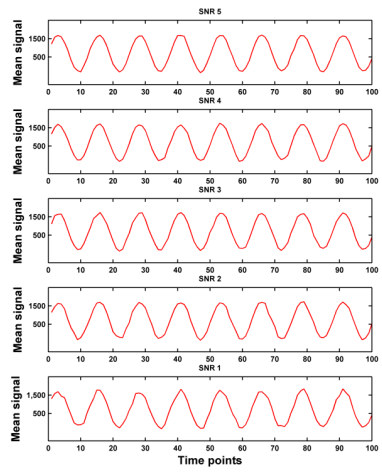

(C) ICA

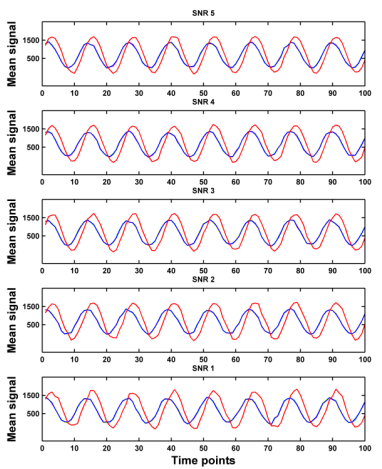

(E) Ncut

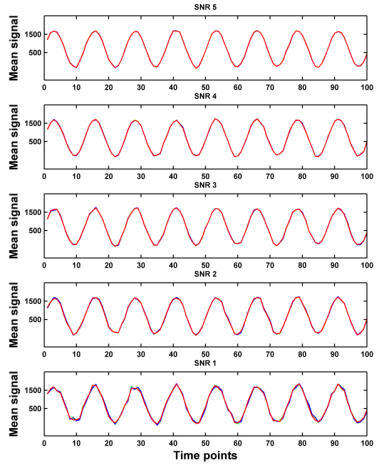

(D) ICA

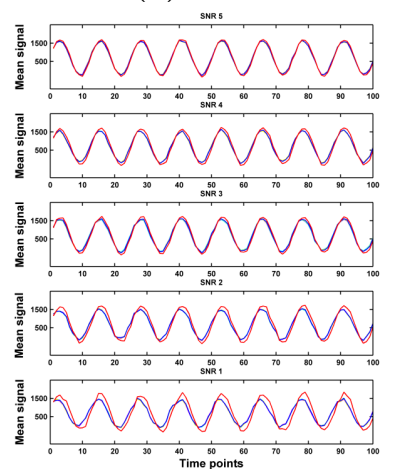

(F) Kmeans

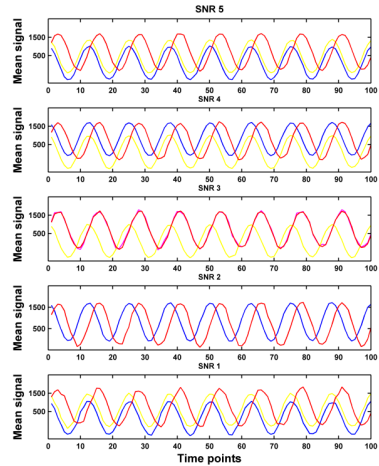

Fig. 4 Signal property under different SNRs, obtained using (A) our method with different $\mathrm{K}$, (B) SCA with different thresholds, (C) ICA with threshold as 2 and different IC numbers, (D) ICA with threshold as 3 and different IC numbers, (E) Ncut with different cluster numbers, and (F) Kmeans with different cluster numbers. Different colors denote different parameters. Related measurement of the simulated network is shown in red.
(A) Our method
(B) SCA

(C) ICA

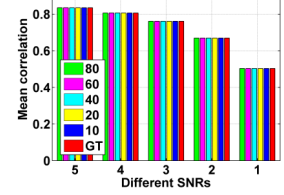

(D) ICA

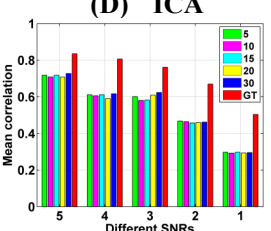

(B) SCA

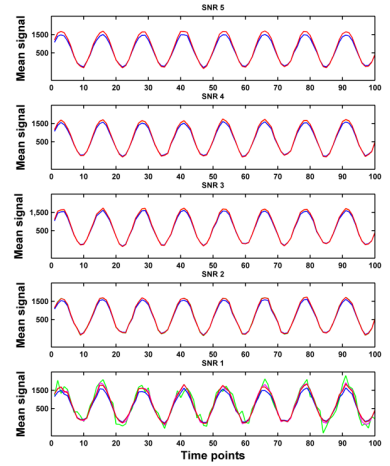

Fig. 5 Network property under different SNRs, obtained using (A) our method with different K, (B) SCA with different thresholds, (C) ICA with threshold as 2 and different IC numbers, (D) ICA with threshold as 3 and different IC numbers, (E) Ncut with different cluster numbers, and (F) Kmeans with different cluster numbers. Different colors denote different parameters. Related measurement of the simulated network (GT) is shown in red.

\subsection{Experiment using resting-state fMRI data}

Resting-state fMRI data of 106 healthy subjects collected at 5 sites were performed by the proposed method to identify subjectspecific default mode network (DMN) and motor network. Firstly, ROIs in posterior cingulate cortex (PCC) and primary motor cortex (M1) were used as seeds of DMN and motor network, respectively. 
Then, voxels that have negative correlation with the mean BOLD time series of those objective seeds were automatically computed as seeds of background. To reduce memory requirements, we used the average BOLD signal in 27 neighbor voxels to represent the signal of a node. Parameter K for constructing k-nearest graph was set to 100 .

Based on the obtained individual network, probability of each voxel was computed as $N / M$, where $M$ denotes the number of all subjects and $N$ denotes the number of subjects whose network includes the voxel. Fig. 6 (A)-(E) show probability maps of DMN across subjects for 5 different sites, respectively. Results indicate that our method can identify subject-specific DMN that had high reliability and reproducibility in important regions, e.g., PCC and anterior cingulate cortex (ACC). For further statistical analysis, for each subject, the Pearson correlation values between one specific voxel and other voxels in the extracted DMN were computed and averaged to reflect the voxel's contribution to the subject-specific network. After obtaining the correlation maps of DMN for all subjects, one-sample t-test with FDR correction of $p$ value $<0.05$ was performed across all subjects of each site, and the results are shown in Fig. 6 (F)-(J), which demonstrate that DMN obtained from different sites had high reproducibility. Similarly, Fig. 7 shows the related results for motor network.

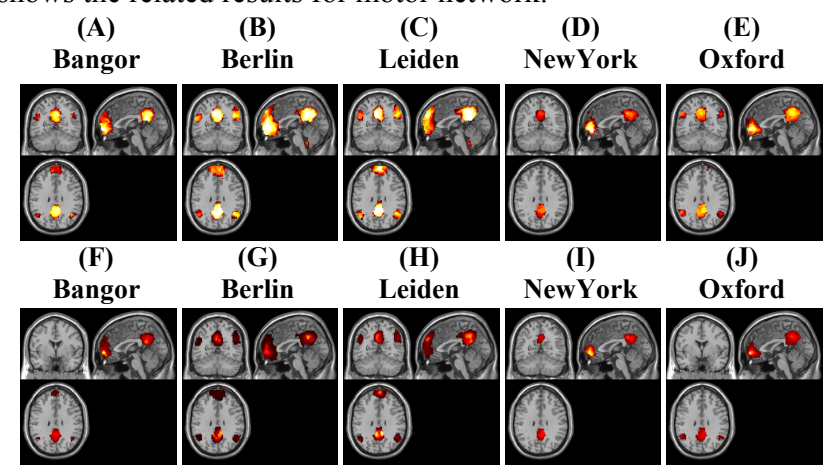

Fig. 6 Results for DMN. (A)-(E): probability map of DMN across subjects from 5 sites, respectively. (F)-(J): one-sample t-test results (FDR correction, $\mathrm{p}$ value $<0.05$ ) of $\mathrm{DMN}$ across subjects for 5 sites, respectively.

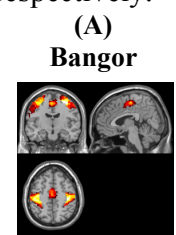

(F)

Bangor

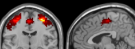

$(5)$

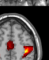

(B)

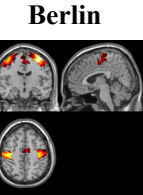

(G)

Berlin

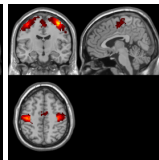

(C)

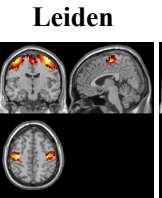

(H) Leiden

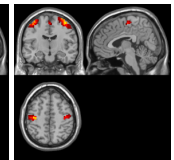

(D)

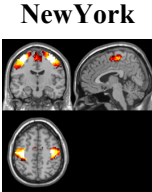

(I)

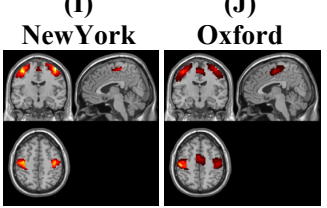

Fig. 7 Results for motor network. (A)-(E): probability map of motor network across subjects for 5 sites, respectively. (F)-(J): one-sample t-test results (FDR correction, $p$ value $<0.05$ ) of motor network across subjects for 5 sites, respectively.

\section{DISCUSSIONS}

To overcome the limitations of existing techniques for functional network extraction, we propose a novel method for identifying subject-specific functional networks of interest based on a semi-supervised learning technique. Experiments using simulated data demonstrate that the proposed method was more robust and accurate than traditional SCA, ICA, and clustering methods including Ncut and Kmeans. Experiments using restingstate fMRI data illustrate that the proposed method can detect subject-specific network, and the extracted networks were reproducible and reliable across subjects and sites. The proposed method showed a better performance due to the incorporation of prior information. In future work, we will also compare the method with other prior-based methods (e.g. spatially constrained ICA) [5]. In addition, other information will be explored for determining seeds, such as Meta-analysis. Other similarity metrics, such as coherence and partial correlation, will be applied to measure the affinity of voxels for extracting network with specific property.

\section{REFERENCES}

[1] K. Li, L. Guo, J. Nie, G. Li, and T. Liu, "Review of methods for functional brain connectivity detection using fMRI," Comput Med Imaging Graph, vol. 33, pp. 131-139, Mar 2009.

[2] B. Biswal, F. Z. Yetkin, V. M. Haughton, and J. S. Hyde, "Functional Connectivity in the Motor Cortex of Resting Human Brain Using Echo-Planar Mri," Magnetic Resonance in Medicine, vol. 34, pp. 537-541, Oct 1995.

[3] V. D. Calhoun, J. Liu, and T. Adali, "A review of group ICA for fMRI data and ICA for joint inference of imaging, genetic, and ERP data," Neuroimage, vol. 45, pp. S163-172, Mar 2009.

[4] V. D. Calhoun and T. Adali, "Multisubject independent component analysis of fMRI: a decade of intrinsic networks, default mode, and neurodiagnostic discovery," IEEE Rev Biomed Eng, vol. 5, pp. 60-73, 2012.

[5] Y. Du and Y. Fan, "Group information guided ICA for fMRI data analysis," Neuroimage, vol. 69, pp. 157-197, Apr 12013.

[6] M. van den Heuvel, R. Mandl, and H. Hulshoff Pol, "Normalized cut group clustering of resting-state FMRI data," PLoS One, vol. 3, p. e2001, 2008.

[7] L. Ferrarini, I. M. Veer, E. Baerends, M. J. van Tol, R. J. Renken, N. J. A. van der Wee, et al., "Hierarchical Functional Modularity in the Resting-State Human Brain," Hum Brain Mapp, vol. 30, pp. 2220-2231, Jul 2009.

[8] D. F. Wang, L. Shi, D. S. Yeung, E. C. C. Tsang, and P. A. Heng, "Ellipsoidal support vector clustering for functional MRI analysis," Pattern Recognition, vol. 40, pp. 2685-2695, Oct 2007.

[9] Y. Du, H. M. Li, H. Wu, and Y. Fan, "Identification of subject specific and functional consistent ROIs using semi-supervised learning," SPIE medical imaging, vol. 83144S, 2012.

[10] X. Zhu, "Semi-supervised learning literature survey," Computer Sciences, 2005.

[11] A. Blum and S. Chawla, "Learning from labeled and unlabeled data using graph mincuts," ICML, 2001.

[12] X. Zhu, Z. Ghahramani, and J. Lafferty, "Semi-supervised learning using gaussian fields and harmonic functions," ICML, 2003.

[13] D. Y. Zhou, O. Bousquet, T. N. Lal, J. Weston, and B. Scholkopf, "Learning with local and global consistency," Advances in Neural Information Processing Systems vol. 16, pp. 321-328, 2004.

[14] J. Wang, T. Jebara, and S. F. Chang., "Graph transduction via alternating minimization," ICML, 2008. 